\title{
Alterstice
}

Revue internationale de la recherche interculturelle

International Journal of Intercultural Research

Revista International de la Investigacion Intercultural

\section{Diffuser la pensée critique malgré tout}

\section{Yvan Leanza}

Volume 5, numéro 2, 2015

URI : https://id.erudit.org/iderudit/1036685ar

DOI : https://doi.org/10.7202/1036685ar

Aller au sommaire du numéro

Éditeur(s)

Alterstice

ISSN

1923-919X (numérique)

Découvrir la revue

Citer ce document

Leanza, Y. (2015). Diffuser la pensée critique malgré tout. Alterstice, 5(2), 1-2.

https://doi.org/10.7202/1036685ar d'utilisation que vous pouvez consulter en ligne.

https://apropos.erudit.org/fr/usagers/politique-dutilisation/ 


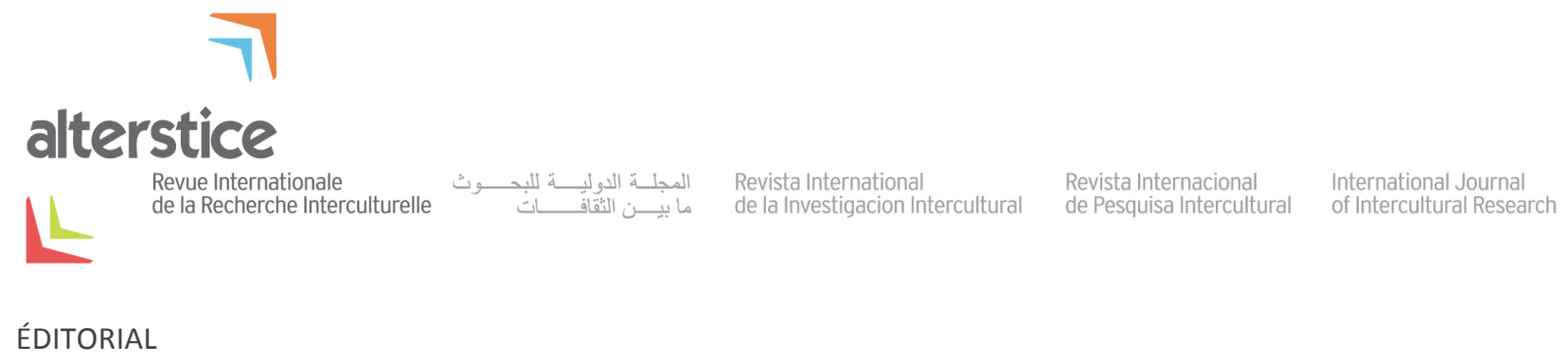

\title{
Diffuser la pensée critique malgré tout
}

\author{
Yvan Leanza $^{1}$
}

Entre l'arrivée continue d'exilés du Moyen-Orient et d'Afrique et les attentats meurtriers, l'Europe, comme le reste du monde, vit des heures sombres. Les simplistes et fanatiques de tous bords, politiques ou religieux, s'en donnent à cœur joie dans ce contexte propice à la réaction impulsive d'autodéfense. Ces réactions viscérales alimentent d'autant plus les sentiments d'exclusion, d'injustice et de dénigrement des groupes visés. Elles font le terreau des simplistes et fanatiques soi-disant de l'autre bord, mais qui, en réalité, partagent la même haine de la différence.

À l'opposé de ces non-débats, la recherche en sciences sociales permet une observation fine de la vie en société doublée d'une remise en question permanente des discours et comportements " de sens commun ". C'est pourquoi, et malgré le triste contexte, je suis particulièrement heureux et fier d'écrire ce dixième éditorial de la revue Alterstice. Voilà maintenant cinq ans et dix numéros que la recherche interculturelle peut employer ce support pour diffuser largement ses résultats et réflexions. Ce champ de recherche met en lumière les universaux du comportement humain et surtout les multiples possibilités d’interprétations, de métissages, de richesses et difficultés des interactions entre personnes de cultures différentes... Une entreprise subversive pour certains, dans le but, je l'espère, de créer plus d'harmonie. Mais n'est-ce là qu'une utopie? C'est peut-être justement ce dont nous avons le plus besoin aujourd'hui : un projet empli d'espoir et d’harmonie, qui va à l'encontre des réponses sécuritaires et militaires peut-être nécessaires, mais limitées dans leur pouvoir de transformation et de résolution de conflit.

Pour célébrer cet anniversaire, le comité éditorial vous offre deux nouveautés. Dès ce numéro, la revue sera diffusée encore plus largement grâce à l'accord établi avec la plateforme Érudit (www.erudit.org). L'intégration à Érudit est une étape importante, puisqu'elle permet aux articles d'être inclus dans des bases de données. Elle ouvre ainsi de nouveaux horizons pour les auteurs. La plateforme s'assure également de la conservation à long terme des documents en employant les services de Portico, le service d'archives sécurisées des presses de l'université Harvard (www.portico.org). Les articles seront référencés dans les agrégateurs suivants, outils de découverte ou de recherche fédérée :

Francis (France); MLA (États-Unis); Ebsco a to z (États-Unis); Primo Central; Meta-lib; Isidore (France); Index savante (France); Synergies (Canada); Base (Allemagne); Journal TOCS (Grande-Bretagne); OAlster (États-Unis); Google scholar (ÉtatsUnis); Proquest; Repère; Summons; World cat.

Ils figureront également dans les bases de données bibliographiques et disciplinaires suivantes :

PubMed; ABCClio; Index Philosopher; FRANCIS (INIST); NINES; Repère; MLA International Bibliography; Arts \& Humanities Citation Index (Web of Science); Social Sciences Citation Index (Web of Science).

La seconde nouveauté est le fruit de l'accord renouvelé entre le comité éditorial et le bureau de l'Association internationale pour la Recherche InterCulturelle (ARIC). Il s'agit d'une nouvelle rubrique, la Rubriqu'ARIC, dans laquelle le bureau de I'ARIC pourra y exprimer son opinion sur les événements interculturels du monde, que cela soit en recherche ou dans les autres sphères de la vie. La première édition de cette rubrique est signée par la présidente de I'ARIC, Michèle Vatz Laaroussi. 
J'ai le grand plaisir d'annoncer ici que le prix ARIC de la meilleure thèse en recherche interculturelle a été remis lors du congrès de l'association à Strasbourg en août 2015. Il s'agit de la thèse intitulée Une approche psychoanthropologique des savoirs à l'" école de la vie " chez des adultes peu ou pas scolarisés au Brésil, soutenue par Candy Laurendon-Marques en décembre 2014, sous la codirection de Annick Weil-Barais, professeure à l'Université d'Angers (France) et de Jorge Tarcisio Da Rocha Falcao, professeur à l'Université fédérale de Rio Grande do Norte (Brésil). L'objectif principal de cette thèse est de rendre compte des conditions d'acquisition des savoirs, essentiellement numériques, à l'« école de la vie " par des adultes brésiliens peu ou pas scolarisés. L'expression "école de la vie " couramment utilisée par les personnes brésiliennes de classe sociale populaire souligne l'importance de la vie quotidienne pour les apprentissages par opposition à l'institution scolaire où ces personnes se trouvent le plus souvent en échec. En adoptant une démarche psycho-anthropologique (observation participante de l'activité quotidienne et professionnelle des adultes, récits de vie), deux études ont été réalisées au Brésil par la lauréate. La première étude concerne les activités quotidiennes de six femmes migrantes issues de la campagne vivant dans la périphérie de Porto Alegre à propos de la gestion du budget et des échanges marchands. La deuxième étude porte sur les pratiques professionnelles d'un commerçant de fruits et légumes suivi pendant six mois, de l'achat des produits à leur vente sur un marché de Recife. Les données ont été analysées à partir du cadre théorique proposé par Barbara Rogoff (1995) qui distingue trois niveaux : institutionnel ou communautaire, inter et intra-personnel. II apparaît que les structures de participation sont très diverses : collaboration avec des proches, quasi-enseignement de la part d'employeur, transmissions de savoir-faire aux générations plus jeunes. Les savoirs numériques sont intriqués dans une constellation de savoirs. Les personnes créent ou adaptent une diversité d'instruments au service de leurs activités.

Le comité éditorial de la revue félicite chaleureusement $\mathrm{M}^{\text {me }}$ Laurendon-Marques et lui souhaite plein succès pour son avenir en recherche interculturelle.

Le thème du présent numéro articule et discute deux notions a priori antithétiques : le patrimoine et l'interculturalité. Sous la direction de l'éditrice invitée, Claire Autant Dorier, de l'Université de Saint-Étienne, ces notions sont déconstruites et reconstruites pour montrer les modalités de faire "patrimoine commun ". Une réflexion nécessaire pour le monde à venir.

Bonne lecture!

\section{Références}

Rogoff, B. (1995). Observing sociocultural activity on three planes: Participatory appropriation, guided participation, and apprenticeship. Dans J. Wertsch, P. del Rio et A. Alvarez (dir.), Sociocultural studies of mind (p. 139-164). Cambridge, UK : Cambridge University Press.

\section{Rattachement de l'auteur}

${ }^{1}$ Université Laval, Québec, Canada

\section{Correspondance}

alterstice@gmail.com

\section{Pour citer cet article}

Leanza, Y. (2015). Diffuser la pensée critique malgré tout [Éditorial]. Alterstice, 5(2), 1-2. 